\title{
Supportive Information
}

\section{Effects of a Thermally Electrochemically Activated ß-PVDF Fiber on Suppression of Li Dendrite Growth for Anode-Free Batteries}

Ljalem Hadush Abrha ${ }^{\mathrm{a}}$, Yosef Nikodimos ${ }^{\mathrm{a}}$, Haile Hisho Weldeyohannes ${ }^{\mathrm{a}}$, Tesfaye Teka Hagos ${ }^{\mathrm{b}}$, DaoYi Wang ${ }^{\mathrm{a}}$, Chen-Jui Huang ${ }^{\mathrm{a}}$, Shi-Kai Jiang ${ }^{\mathrm{a}}$, She-Huang $\mathrm{Wu}^{\mathrm{b}}$, Wei-Nien Su${ }^{\mathrm{b} *}$, Meng-Che Tsai ${ }^{\mathrm{b}}$, Bing Joe Hwang ${ }^{\mathrm{a}, \mathrm{c}, \mathrm{d} *}$

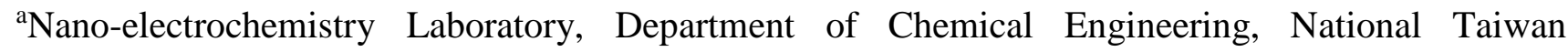
University of Science and Technology, Taipei 106, Taiwan

${ }^{b}$ Nano-electrochemistry Laboratory, Graduate Institute of Applied Science and Technology, National Taiwan University of Science and Technology, Taipei 106, Taiwan

'Sustainable Energy Development Center, National Taiwan University of Science and Technology, Taipei, 106, Taiwan

${ }^{\mathrm{d} N a t i o n a l ~ S y n c h r o t r o n ~ R a d i a t i o n ~ R e s e a r c h ~ C e n t e r, ~ H s i n-C h u ~ 30076, ~ T a i w a n ~}$ 

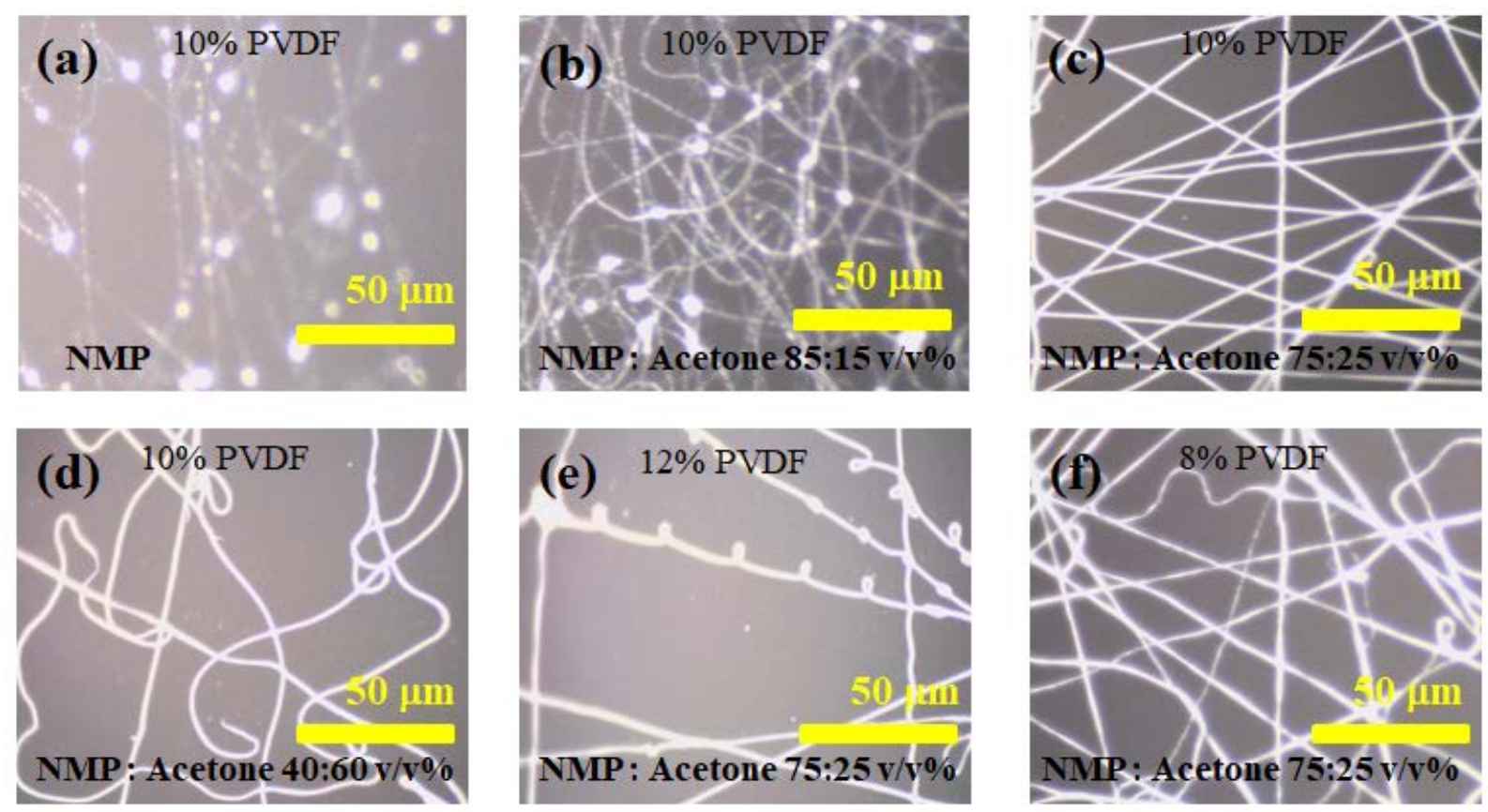

Figure S1. OM images of $\beta$-PVDF fibers coated Cu prepared with various solvents ratio and PVDF concentrations (a) 100\% NMP solvent with 10\% PVDF, (b) NMP : Acetone (85:15 v/v\%) with 10\% PVDF, (c) NMP : Acetone (75:25 v/v\%) with 10\% PVDF, (d) NMP : Acetone (40:60 v/v\%) with 10\% PVDF, (e) NMP: Acetone (75:25 v/v\%) with 12\% PVDF, and (f) NMP: Acetone (75:25 v/v\%) with 8\% PVDF. 

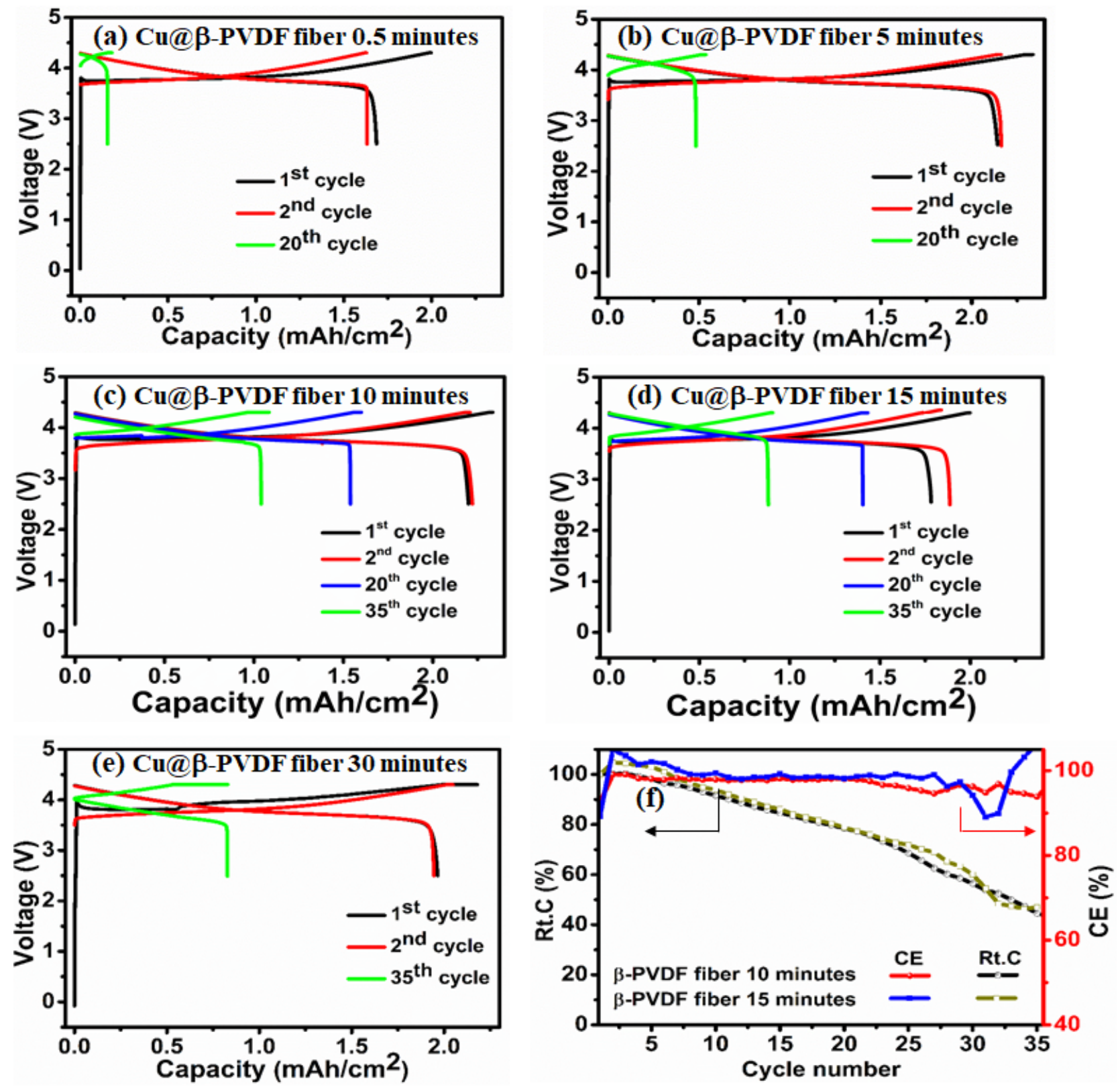

Figure S2. Charge/discharge curves of the selected cycles of $\mathrm{Cu@ \beta -PVDF \| NMC} \mathrm{cells} \mathrm{assembled} \mathrm{with}$ $\beta$-PVDF fiber modified Cu by electrospinning coating for (a) 0.5 minutes (0.5'), (b) 5 minutes (5'), (c) 10 minutes (10'), (d) 15 minutes (15'), and (e) 30 minutes (30') at current density of $0.2 \mathrm{~mA} \mathrm{~cm}^{-2}$, (f) retention capacity and coulombic efficiencies of the cells prepared with fiber modified $\mathrm{Cu}$ by electrospun-coating for $10^{\prime}$ and 15 ' in a potential window range of $(2.5-4.3 \mathrm{~V})$. 

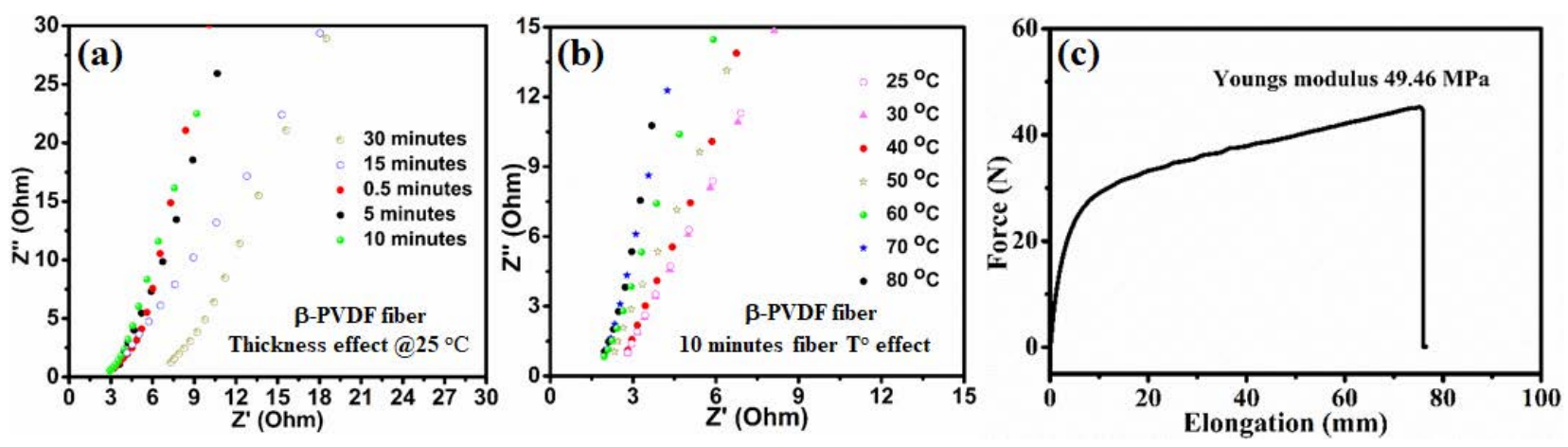

Figure S3. $\mathrm{Cu} @ \beta-\mathrm{PVDF} \| \mathrm{Cu} @ \beta$-PVDF symmetric cells prepared with electrolyte of $1 \mathrm{M} \mathrm{LiPF}_{6}$ in EC/DEC (1:1 v/v) for cells prepared with (a) $0.5,5,10,15$, and 30 minutes' electrospun-coated fiber at $25^{\circ} \mathrm{C}$ (b) 10 minutes' electrospun-coated $\mathrm{Cu}$ at temperatures range between 25 and $80{ }^{\circ} \mathrm{C}$ performed within frequency range of $0.1 \mathrm{~Hz}-100 \mathrm{kHz}$ (c) modulus of PVDF polymer. 

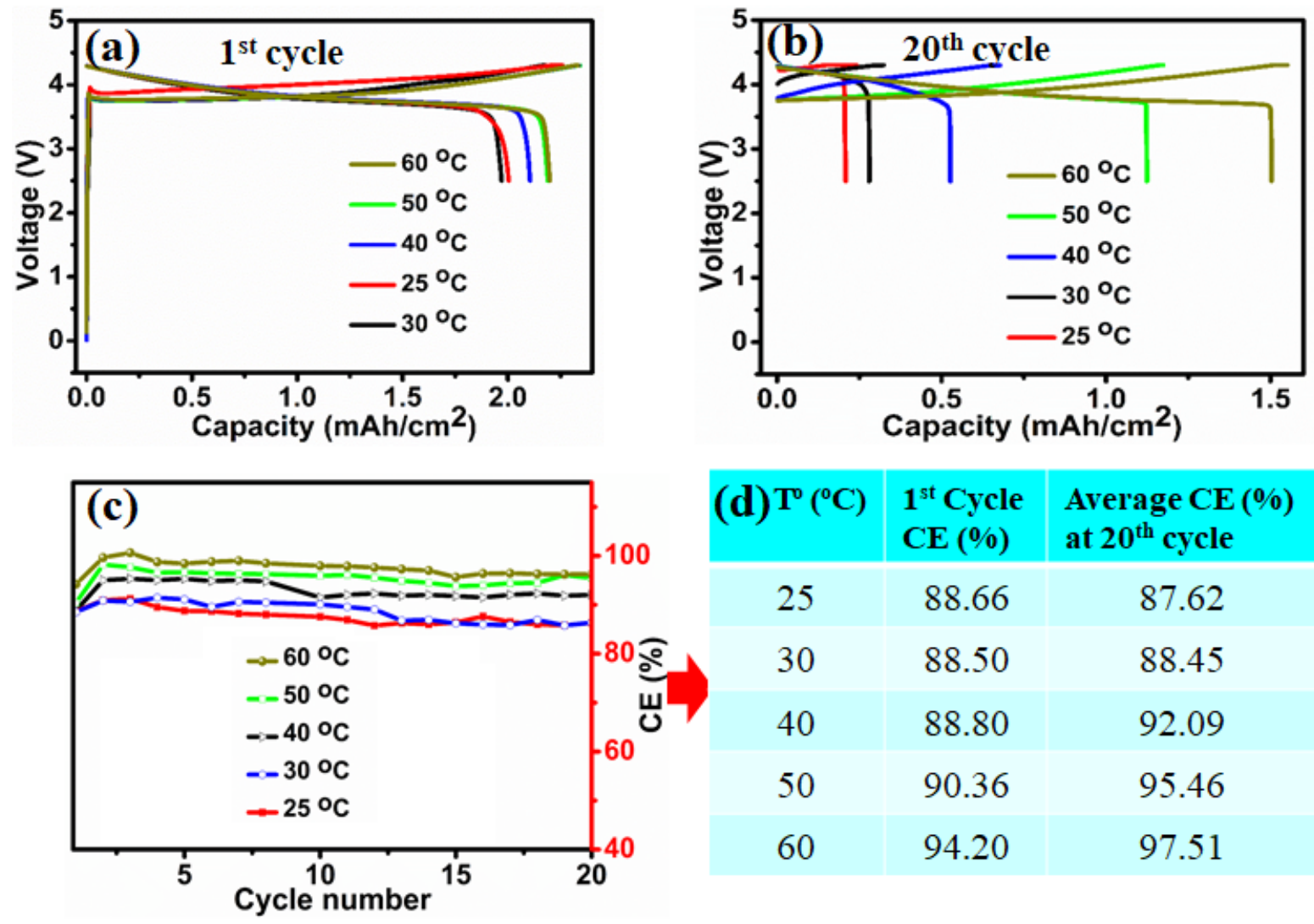

\begin{tabular}{|c|c|c|}
\hline$(\mathrm{d}) \mathrm{T}^{\mathrm{T}}\left({ }^{0} \mathrm{C}\right)$ & $\begin{array}{l}1^{\text {st }} \text { Cycle } \\
\text { CE (\%) }\end{array}$ & $\begin{array}{l}\text { Average CE }(\%) \\
\text { at } 20^{\text {th }} \text { cycle }\end{array}$ \\
\hline 25 & 88.66 & 87.62 \\
\hline 30 & 88.50 & 88.45 \\
\hline 40 & 88.80 & 92.09 \\
\hline 50 & 90.36 & 95.46 \\
\hline 60 & 94.20 & 97.51 \\
\hline
\end{tabular}
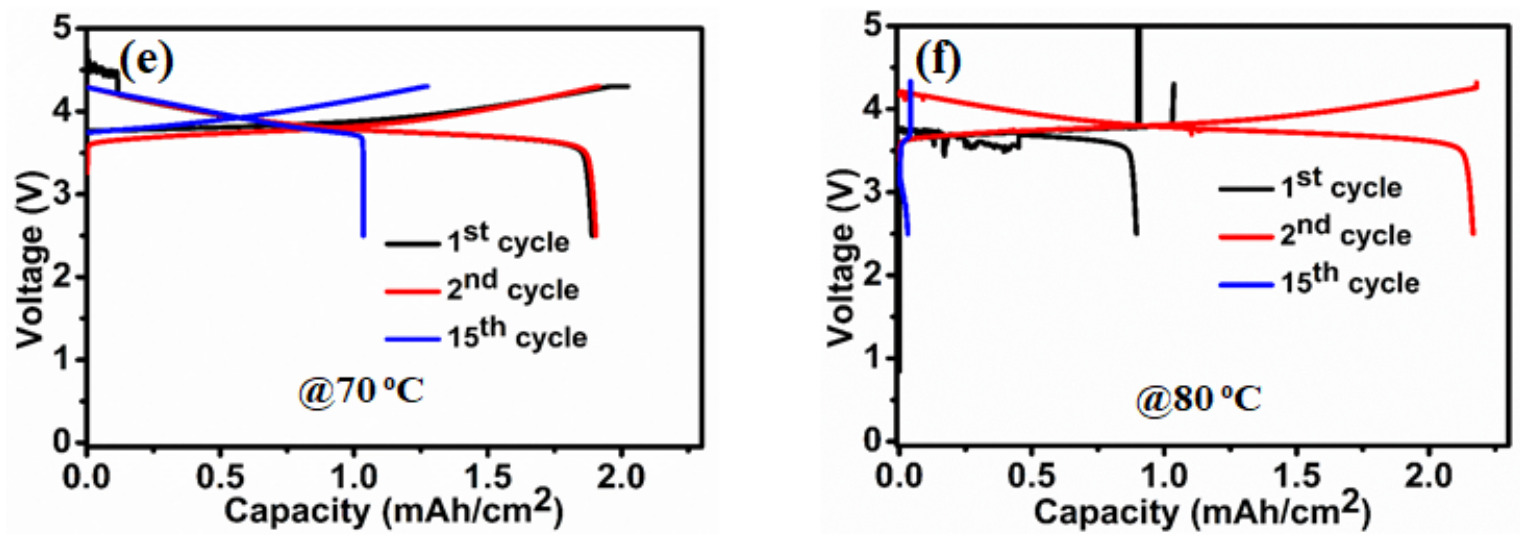

Figure S4. Charge/discharge curves of Cu@ $\beta-P V D F \| N M C$ cell (a) 1st cycle (b) $20^{\text {th }}$ cycle (c) capacity retention and coulombic efficiency (CE) versus cycle number of cells prepared with 10' electrospinning-coated $\mathrm{Cu}$ electrode at temperatures of $25-60{ }^{\circ} \mathrm{C}$ (d) cycle number versus average $\mathrm{CE}$ of selected cycles (e, f) charge/discharge curves of $70,80^{\circ} \mathrm{C}$ were performed at a current density of 0.2 $\mathrm{mA} \mathrm{cm}{ }^{-2}(2.5-4.3 \mathrm{~V})$. 


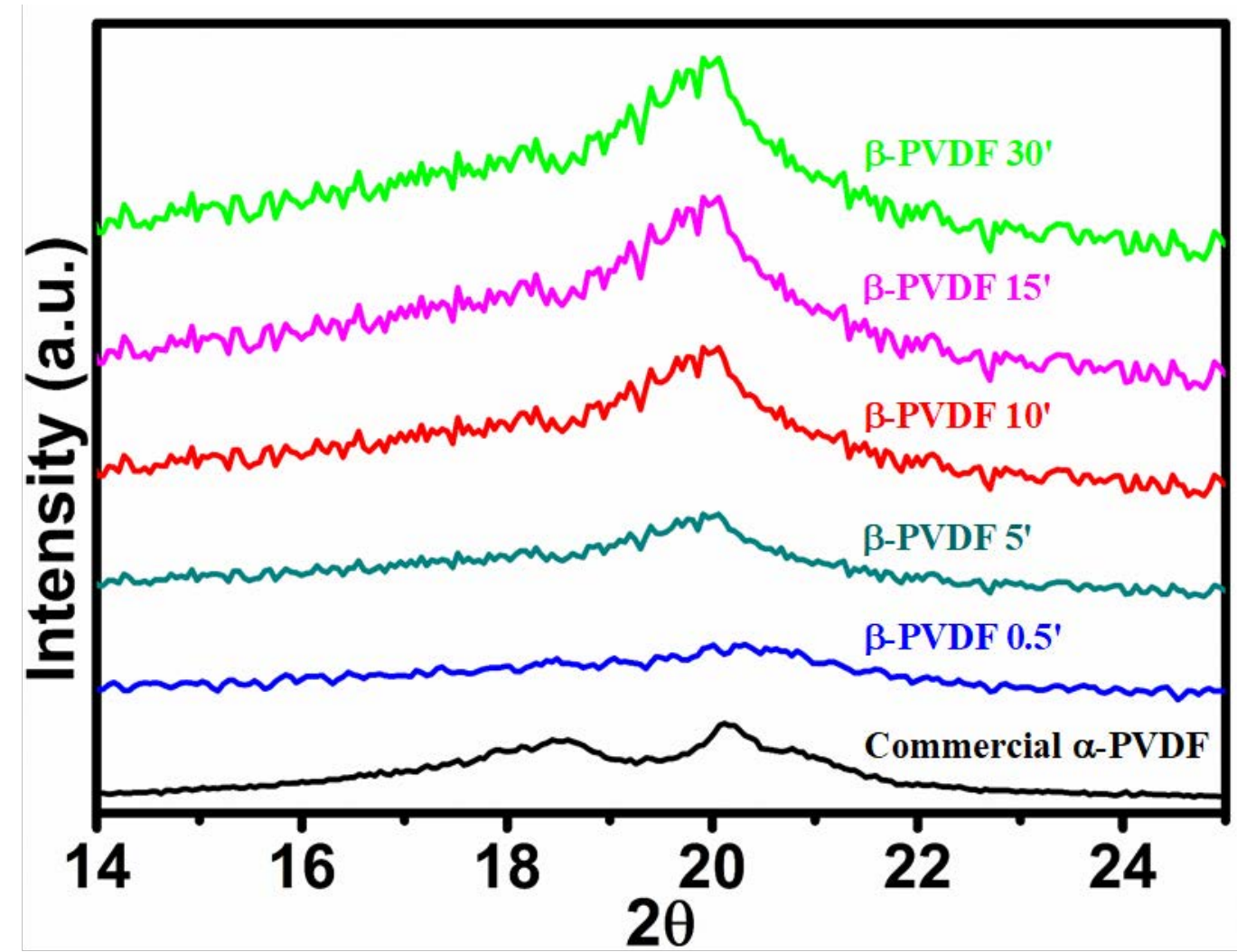

Figure S5. XRD patterns of commercial $\alpha$-PVDF, 0.5, 5, 10, 15, and 30 minutes (30') $\beta$-PVDF electrospun deposited fibers. 


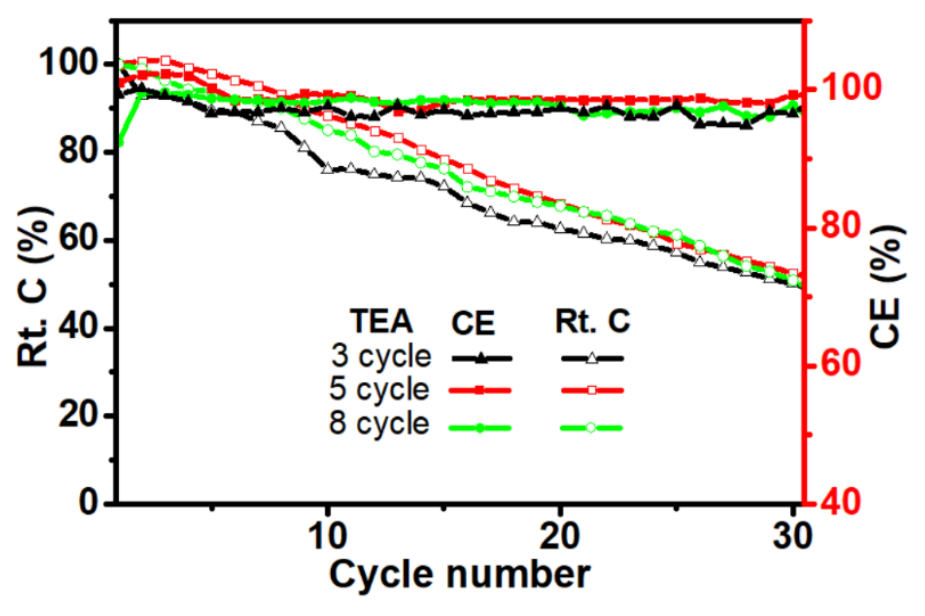

Figure S6. Capacity retentions of $\mathrm{Cu} @ \beta-\mathrm{PVDF} \| \mathrm{NMC}$ cells performed at $25^{\circ} \mathrm{C}$ after TEA of 3, 5, 8 cycles.

Table S1. Comparisons of Coulombic efficiency, capacity retention versus cycle number between $\mathrm{Cu} @ \alpha-\mathrm{PVDF} \| \mathrm{NMC}$ and $\mathrm{Cu} @ \beta-\mathrm{PVDF} \| \mathrm{NMC}$ at different charge/discharge operation temperature $\left(\mathrm{T}^{\circ}\right)$.

\begin{tabular}{ccccc}
\hline $\begin{array}{c}\text { Charge/discharge } \\
\text { operation temperature }\end{array}$ & Type of PVDF & $1^{\text {st }}$ CE (\%) & \multicolumn{2}{c}{$20^{\text {th }}$ cycle } \\
\hline $25{ }^{\circ} \mathrm{C}$ & $\alpha-P V D F$ & 84.64 & 87.22 & 9.22 \\
& $\beta$-PVDF & 88.66 & 87.62 & 12.43 \\
\hline TA $25{ }^{\circ} \mathrm{C}$ & $\beta$-PVDF & 96.64 & 91.77 & 22.69 \\
\hline TEA $60{ }^{\circ} \mathrm{C}$ & $\alpha-P V D F$ & 93.38 & 95.56 & 35.36 \\
& $\beta$-PVDF & 94.20 & 97.51 & 78.45 \\
\hline TEA $25{ }^{\circ} \mathrm{C}$ & $\alpha-P V D F$ & 92.38 & 94.46 & 37.43 \\
& $\beta-P V D F$ & 95.92 & 99.22 & 68.36 \\
\hline
\end{tabular}



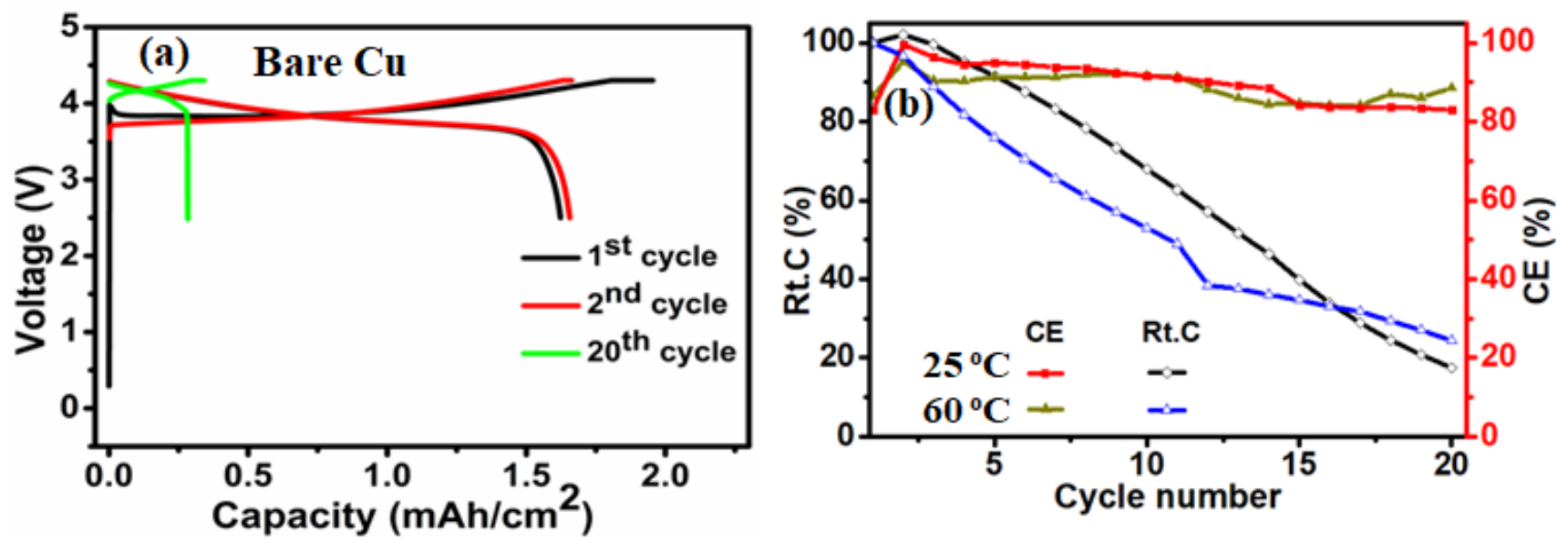

Figure S7. Charge/discharge curves of (a) bare $\mathrm{Cu} \| \mathrm{NMC}$ performed at $25{ }^{\circ} \mathrm{C}$ (b) capacity retention versus coulombic efficiencies performed at $25,60{ }^{\circ} \mathrm{C}\left(0.2 \mathrm{~mA} \mathrm{~cm}{ }^{-2}\right)$.
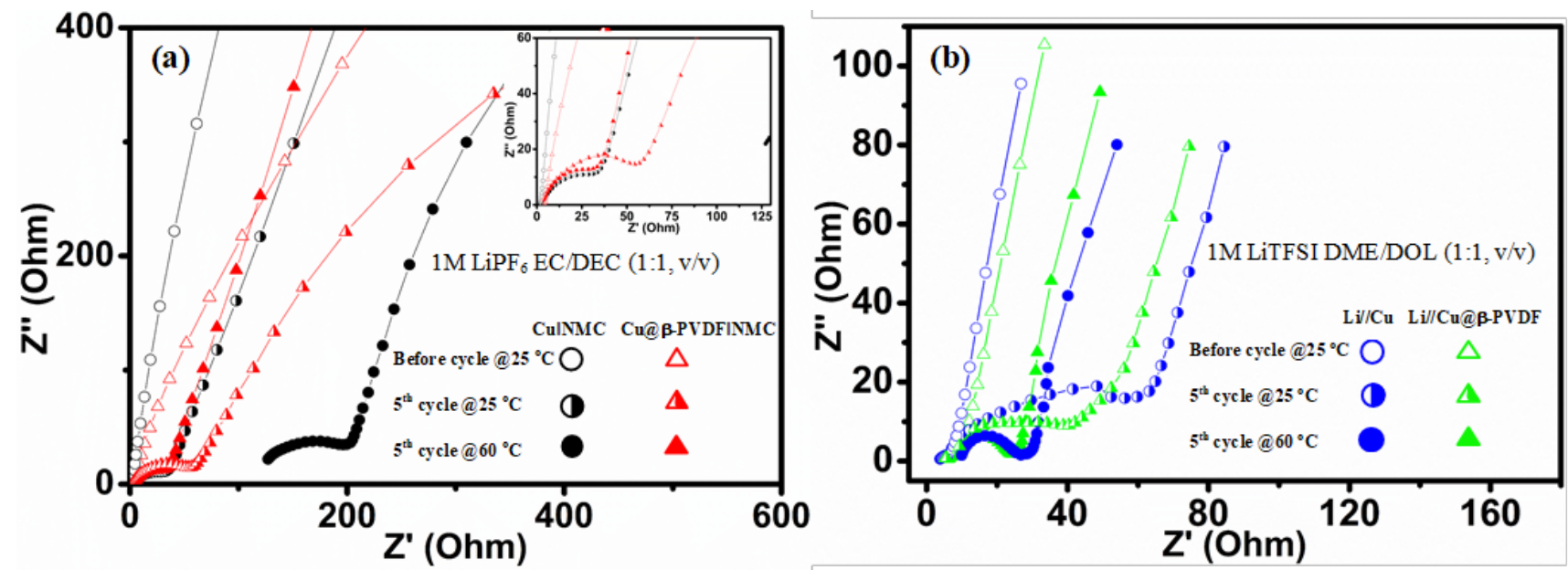

Figure S8. Nyquist plots of (a) Cu\|NMC in comparison with Cu@ $\beta-P V D F \| N M C$ using 1M LiPF 6 EC/DEC (1:1 v/v) electrolyte (b) Li//Cu versus Li//Cu@ $\beta$-PVDF cell tested at 25 and $60{ }^{\circ} \mathrm{C}$ using $1 \mathrm{M}$ LiTFSI DME/DOL electrolyte measured before cycle, at $5^{\text {th }}$ cycle in a frequency range of $0.1 \mathrm{~Hz}-100$ $\mathrm{kHz}$. 
Table S2. Fitting results EIS of both CulNMC, Cu@ $\beta-P V D F \| N M C$ using 1M LiPF 6 EC/DEC (1:1 v/v) electrolyte and Li//Cu, Li//Cu@ $\beta$-PVDF using 1M LiTFSI DME/DOL before, and after 5 cycles charged/discharged at $0.2 \mathrm{~mA} / \mathrm{cm}^{2}$ (at 25,60$){ }^{\circ} \mathrm{C}$ operation temperatures.

\begin{tabular}{|c|c|c|c|c|}
\hline Cell type & $\mathrm{T}^{0}\left({ }^{\circ} \mathrm{C}\right)$ & $\mathrm{R}_{\mathrm{s}}(\mathrm{Ohm})$ & $\mathrm{R}_{\text {sei }}(\mathrm{Ohm})$ & $\mathrm{R}_{\mathrm{ct}}(\mathrm{Ohm})$ \\
\hline \multirow{3}{*}{$\begin{array}{l}\mathrm{Cu} \| \mathrm{NMC} \\
(1 \mathrm{M} \mathrm{LiPF} 6 \mathrm{EC} / \mathrm{DEC})\end{array}$} & Before cycle $25^{\circ} \mathrm{C}$ & 3.02 & 10.05 & 7.03 \\
\hline & $5^{\text {th }}$ cycle $25^{\circ} \mathrm{C}$ & 3.58 & 26.29 & 22.71 \\
\hline & $5^{\text {th }}$ cycle $60^{\circ} \mathrm{C}$ & 6.03 & 206.15 & 200.12 \\
\hline \multirow{3}{*}{$\begin{array}{l}\mathrm{Cu} @ \beta-P V D F \| N M C \\
(1 \mathrm{M} \mathrm{LiPF} 6 \text { EC/DEC) }\end{array}$} & Before cycle $25^{\circ} \mathrm{C}$ & 3.91 & 11.91 & 8.00 \\
\hline & $5^{\text {th }}$ cycle $25^{\circ} \mathrm{C}$ & 4.60 & 56.60 & 52.00 \\
\hline & $5^{\text {th }}$ cycle $60^{\circ} \mathrm{C}$ & 5.76 & 25.05 & 22.10 \\
\hline $\mathrm{Li} / / \mathrm{Cu}$ & Before cycle $25^{\circ} \mathrm{C}$ & 3.44 & 7.18 & 3.74 \\
\hline (1M LiTFSI & $5^{\text {th }}$ cycle $25^{\circ} \mathrm{C}$ & 5.72 & 63.49 & 56.71 \\
\hline DME/DOL) & $5^{\text {th }}$ cycle $60^{\circ} \mathrm{C}$ & 6.03 & 31.23 & 25.01 \\
\hline Li//Cu@ $\beta-P V D F$ & Before cycle $25^{\circ} \mathrm{C}$ & 5.92 & 9.23 & 3.61 \\
\hline (1M LiTFSI & $5^{\text {th }}$ cycle $25^{\circ} \mathrm{C}$ & 5.58 & 41.01 & 35.13 \\
\hline DME/DOL) & $5^{\text {th }}$ cycle $60^{\circ} \mathrm{C}$ & 5.76 & 25.05 & 19.29 \\
\hline
\end{tabular}



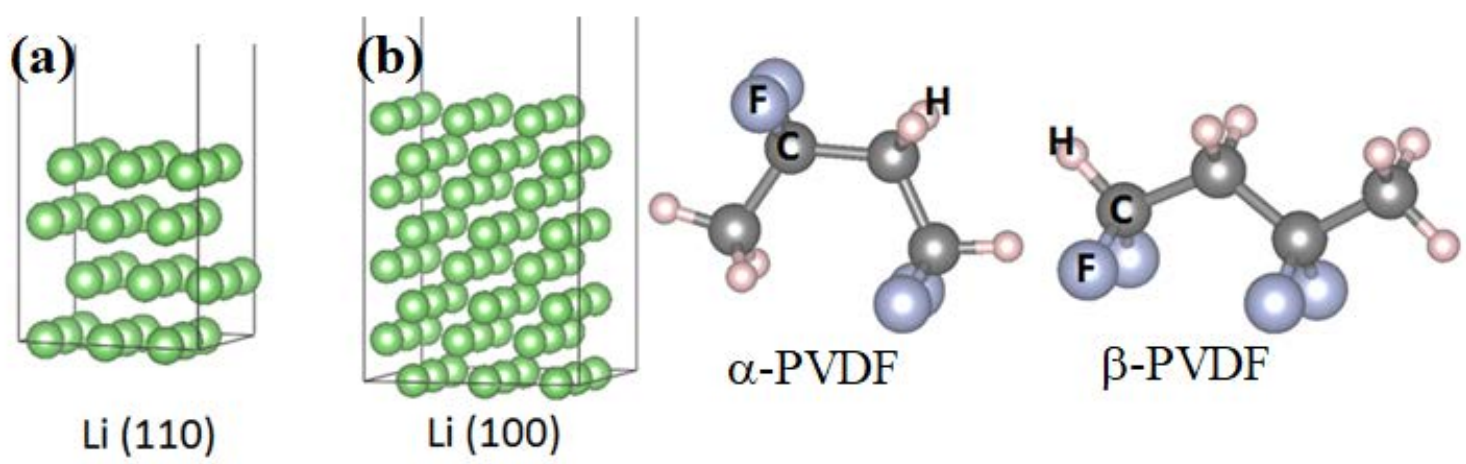

Li (110)
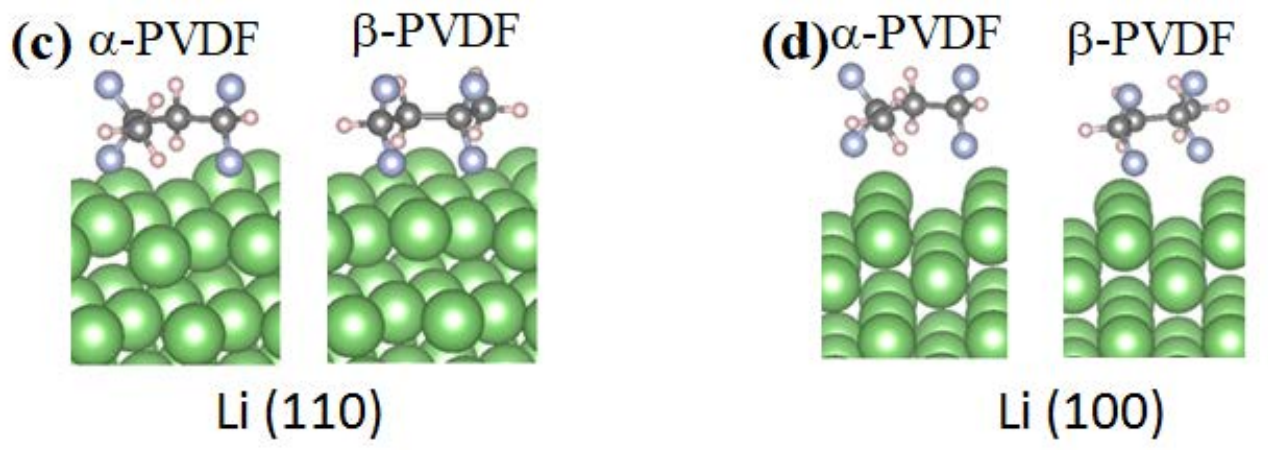

Figure S9. Adsorption models of $\alpha$ and $\beta$-PVDF fiber on Li surface.

Table S3. Simulation adsorption energy of $\alpha$ and $\beta$-PVDF fiber on Li surface.

\begin{tabular}{ccc}
\hline Li Surfaces & Adsorbates & $\boldsymbol{E}_{\text {ads }} / \mathbf{e V}$ \\
\hline $\operatorname{Li}(100)$ & $\alpha-P V D F$ & +0.03 \\
& $\beta-P V D F$ & -0.09 \\
$\operatorname{Li}(110)$ & $\alpha-P V D F$ & +0.07 \\
& $\beta-P V D F$ & -0.05 \\
\hline
\end{tabular}



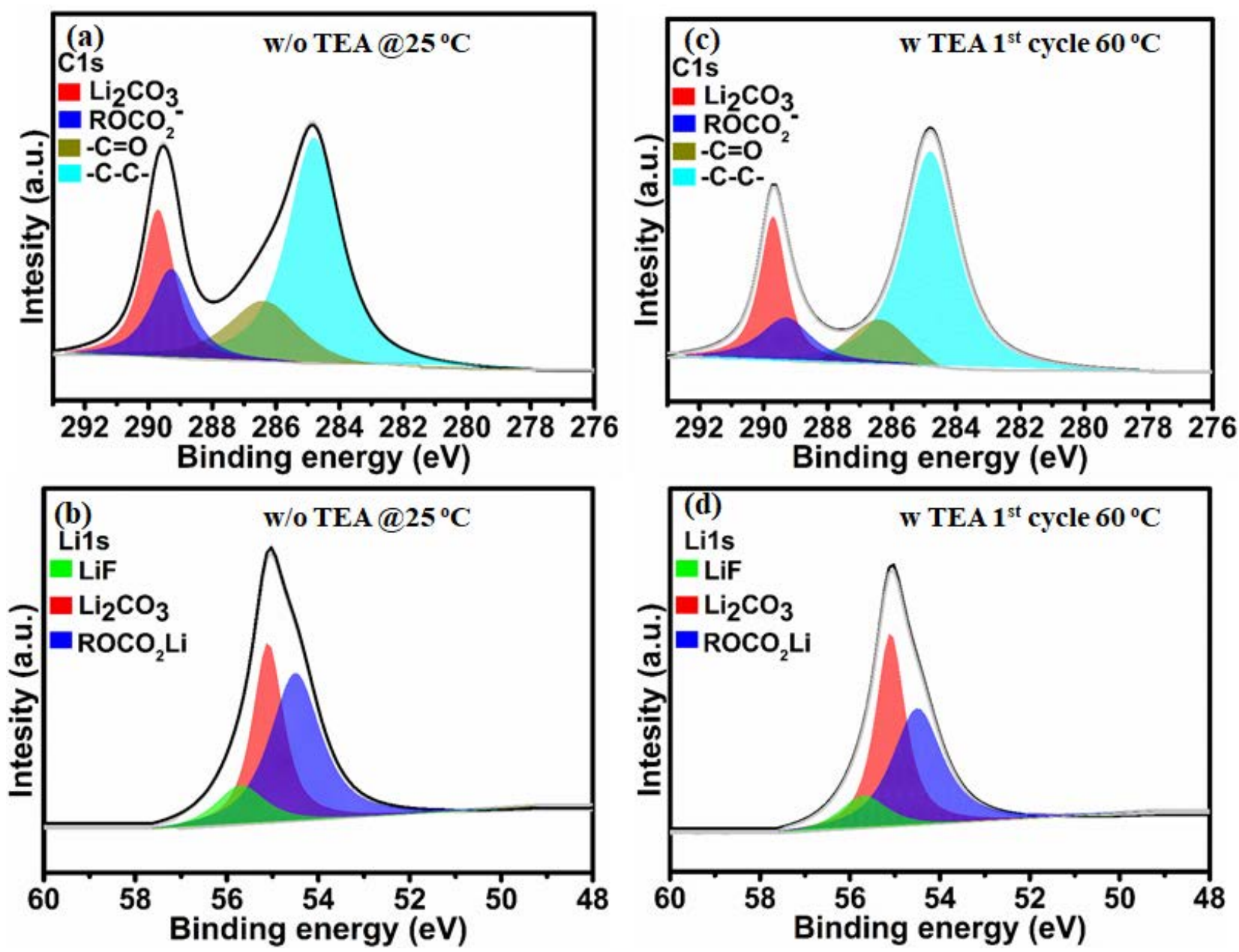

Figure S10. C1s and Li1s XPS spectra of Cu@ $\alpha$-PVDF fiber (a, b) w/o TEA $25{ }^{\circ} \mathrm{C}(\mathrm{c}, \mathrm{d}) \mathrm{w}$ TEA $1^{\text {st }}$ cycle at $60{ }^{\circ} \mathrm{C}\left(0.2 \mathrm{~mA} \mathrm{~cm}^{-2}\right.$ current density). 

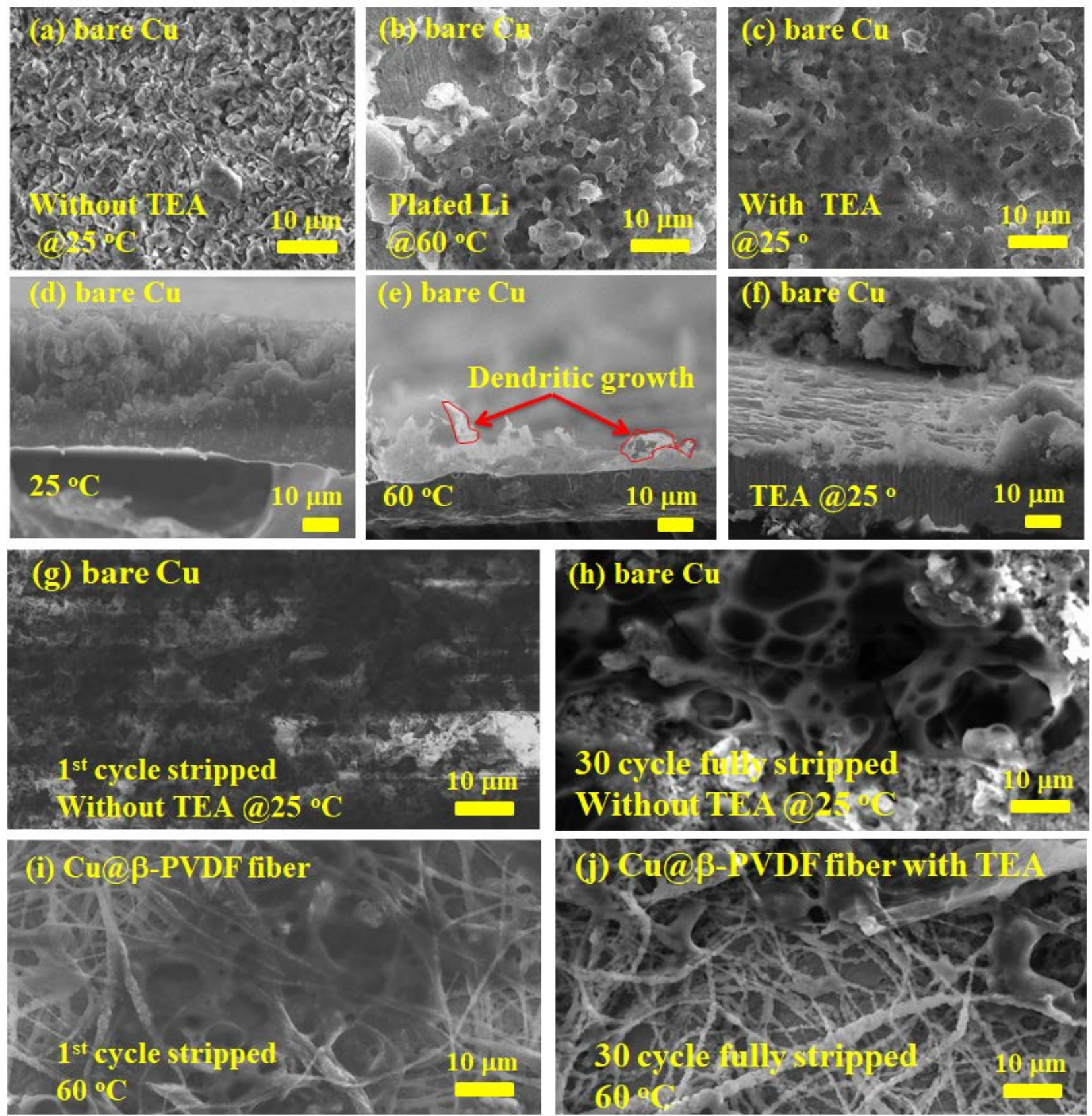

Figure S11. Top view and cross sectional SEM of lithium plated on bare $\mathrm{Cu}$ at (a-f) $25{ }^{\circ} \mathrm{C}, 60{ }^{\circ} \mathrm{C}$ without and with TEA respectively $(\mathrm{g}, \mathrm{h})$ top view morphology on bare $\mathrm{Cu}$ at $1^{\text {st }}, 30$ cycle stripped (i, j) on Cu@ $\beta$-PVDF fiber after $1^{\text {st }}, 30$ cycle fully stripped at a current density of $0.2 \mathrm{~mA} \mathrm{~cm}{ }^{-2}$ performed at $60{ }^{\circ} \mathrm{C}$. 

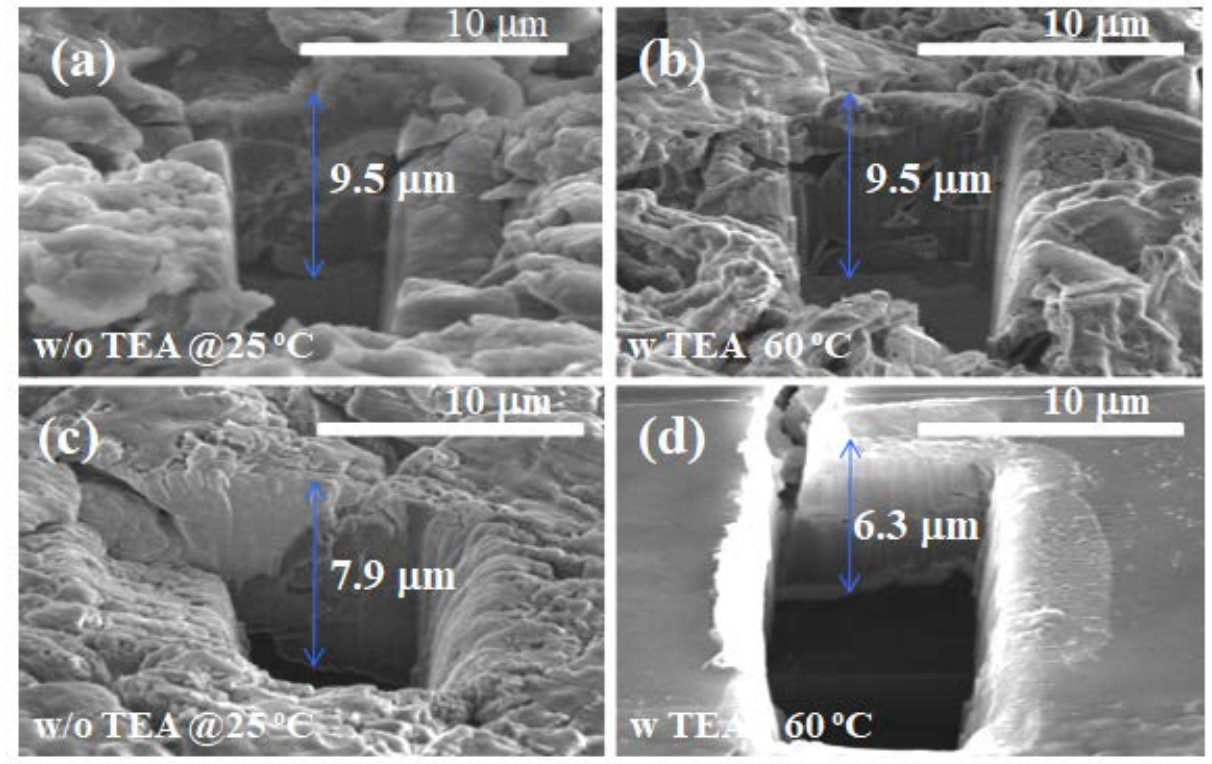

Figure S12. FIB SEM images of Li plating at the initial cycle on bare $\mathrm{Cu}$ (a) without TEA at $25^{\circ} \mathrm{C}$ (b) with TEA at $60{ }^{\circ} \mathrm{C}$, and on $\mathrm{Cu} @ \beta$-PVDF fiber (c) without TEA at $25{ }^{\circ} \mathrm{C}$ (d) with TEA at $60{ }^{\circ} \mathrm{C}$ at a current density of $0.2 \mathrm{~mA} \mathrm{~cm}^{-2}$. 